Please do not remove this page

RMIT

UNIVERSITY

\title{
Stretchable and Tunable Microtectonic ZnO-Based Sensors and Photonics
}

Gutruf, Philipp Jonas; Zeller, Eike; Walia, Sumeet; Nili Ahmadabadi, Hussein; Sriram, Sharath; Bhaskaran, Madhu

https://researchrepository.rmit.edu.au/esploro/outputs/9921862547901341/filesAndLinks?institution=61RMIT_INST\&index=null

Gutruf, P. J., Zeller, E., Walia, S., Nili Ahmadabadi, H., Sriram, S., \& Bhaskaran, M. (2015). Stretchable and Tunable Microtectonic ZnO-Based Sensors and Photonics. Small, 11(35), 4532-4539.

https://doi.org/10.1002/smll.201500729

Document Version: Accepted Manuscript

Published Version: https://doi.org/10.1002/smll.201500729

Repository homepage: https://researchrepository.rmit.edu.au

(c) 2015 WILEY-VCH Verlag GmbH \& Co. KGaA, Weinheim.

Downloaded On 2023/04/27 00:03:20 +1000 
Thank you for downloading this document from the RMIT Research Repository.

The RMIT Research Repository is an open access database showcasing the research outputs of RMIT University researchers.

RMIT Research Repository: http://researchbank.rmit.edu.au/

\section{Citation:}

Gutruf, P, Zeller, E, Walia, S, Nili Ahmadabadi, H, Sriram, S and Bhaskaran, M 2015, 'Stretchable and tunable microtectonic ZnO-based sensors and photonics', Small, vol. 11, no. 35, pp. 4532-4539.

See this record in the RMIT Research Repository at:

https://researchbank.rmit.edu.au/view/rmit:33078

Version: Accepted Manuscript

Copyright Statement:

(C) 2015 WILEY-VCH Verlag GmbH \& Co. KGaA, Weinheim

\section{Link to Published Version:}

https://dx.doi.org/10.1002/smll.201500729 


\section{WILEY-VCH}

DOI: 10.1002/smll.((please add manuscript number))

Article type: Full Paper

\section{Stretchable and tunable micro-tectonic ZnO-based sensors and photonics}

By Philipp Gutruf, Eike Zeller, Sumeet Walia, Hussein Nili, Sharath Sriram,* and Madhu Bhaskaran*

[*] Mr. Philipp Gutruf, Dr. Eike Zeller, Dr. Sumeet Walia, Mr. Hussein Nili, Dr. Sharath Sriram, Dr. Madhu Bhaskaran

Functional Materials and Microsystems Research Group and Micro Nano Research Facility, RMIT University, Melbourne, Victoria (Australia)

E-mail: sharath.sriram@rmit.edu.au,madhu.bhaskaran@rmit.edu.au

Keywords: stretchable electronics, ZnO, gas sensing, UV sensing, stretchable gratings

The concept of realizing electronic applications on elastically stretchable 'skins' that conform to irregularly-shaped surfaces is revolutionizing fundamental research into mechanics and materials that can enable high performance stretchable devices. The ability to operate electronic devices under various mechanically-stressed states can provide a set of unique functionalities that are beyond the capabilities of conventional rigid electronics. Here, we introduce a distinctive micro-tectonic effect enabled oxygen-deficient, nano-patterned zinc oxide $(\mathrm{ZnO})$ thin films on an elastomeric substrate to realize large area, stretchable, transparent, and ultra-portable sensors. The unique surface structures are exploited to create stretchable gas and ultra-violet light sensors, where the functional oxide itself is stretchable, both of which outperform their rigid counterparts under room temperature conditions. Nanoscale $\mathrm{ZnO}$ features embedded in an elastomeric matrix function as tunable diffraction gratings, capable of sensing displacements with nanometre accuracy. These devices and the micro-tectonic oxide thin film approach show promise in enabling functional, transparent, and wearable electronics. 


\section{WILEY-VCH}

\section{Introduction}

The incorporation of electronic materials, in particular functional metal oxides onto mechanically conformal platforms offers a new pathway for the development of sophisticated, stretchable electronic devices that can be bent, stretched, twisted, and folded into complex curvilinear shapes while maintaining their electronic characteristics, performance, and reliability. This new class of electronics is promising for designing novel systems, such as in vitro $\mathrm{pH}$ sensors, ${ }^{[1]}$ transient ${ }^{[2]}$ and printable electronic devices, ${ }^{[3]}$ sensory robotic skin, and wearable electronic devices.

Owing to rapid development in recent years, some flexible devices have been demonstrated to operate almost at par with their rigid counterparts in consumer applications such as organic LEDs, ${ }^{[4]}$ stretchable displays, ${ }^{[5,6]}$ and high-speed transistors, ${ }^{[7]}$ as well as devices operating on the epidermis. ${ }^{[8]}$ However, the wide reach of this technology is yet to be fully exploited. A potentially futuristic vision could be the realization of a completely autonomous sensing platform such as a flexible devices based on 'smart-dust'. ${ }^{[9]}$ The realization of such systems will rely on designing electronic devices that are lightweight, stretchable, and transparent. Metal oxides are attractive candidates as the functional materials for a variety of such electronic and optoelectronic applications. Their electronic properties can range from insulating to semiconducting and can be readily tuned by morphological and stoichiometric alterations. ${ }^{[10]}$ Additionally, their carrier transport properties are well-understood and various engineering processes are well-established for manipulating them. The prospect of incorporating them on flexible platforms offers a potential for designing a wide variety of tunable, stretchable sensing platforms (with the added benefit of optical transparency or translucency). 


\section{WILEY-VCH}

In this work, we demonstrate the integration of a multifunctional metal oxide (zinc oxide, $\mathrm{ZnO}$ ) on a prevalent, biocompatible, flexible substrate, polydimethylsiloxane (PDMS). ZnO is chosen as the representative functional oxide due to well-established versatility of its electronic and optical characteristics, susceptibility to modulation via a variety of stimuli, and bio-compatibility. We exploit the micro-tectonic surface structure of the $\mathrm{ZnO}$ thin films that is uniquely obtained on flexible substrates, to demonstrate high performance sensing devices. The gas sensors demonstrated in this work are able to sense hazardous environments (explosive pollutant and toxic gases such as $\mathrm{H}_{2}$ and $\mathrm{NO}_{2}$ gases even at extremely low concentrations) that can occur in domestic or industrial settings. We also establish their capability for ultra-violet (UV) radiation sensing and demonstrate diffraction gratings that are able to sense strain with nanometre resolution.

To overcome the technological difficulties that are encountered in the fabrication of such devices, we implemented a transfer technique presented in our previous work. ${ }^{[1]}$ The process relies on the poor adhesion of platinum to silicon which allows high temperature oxide thin films to be deposited and defined with standard micro fabrication techniques and subsequently peeled-off using PDMS. This process was adapted for $\mathrm{ZnO}$ and for nanoscale features, as depicted in Figure 1. This ubiquitous technique allows the creation of transparent stretchable electronics with nanometre resolution as well as large area functional devices without the need of tailoring the production process to the design. The large area device without electrodes is shown as a photographic picture in Figure 2a, while the micro-tectonic morphology of the $\mathrm{ZnO}$ surface is shown in Figure 2b. 


\section{WILEY-VCH}

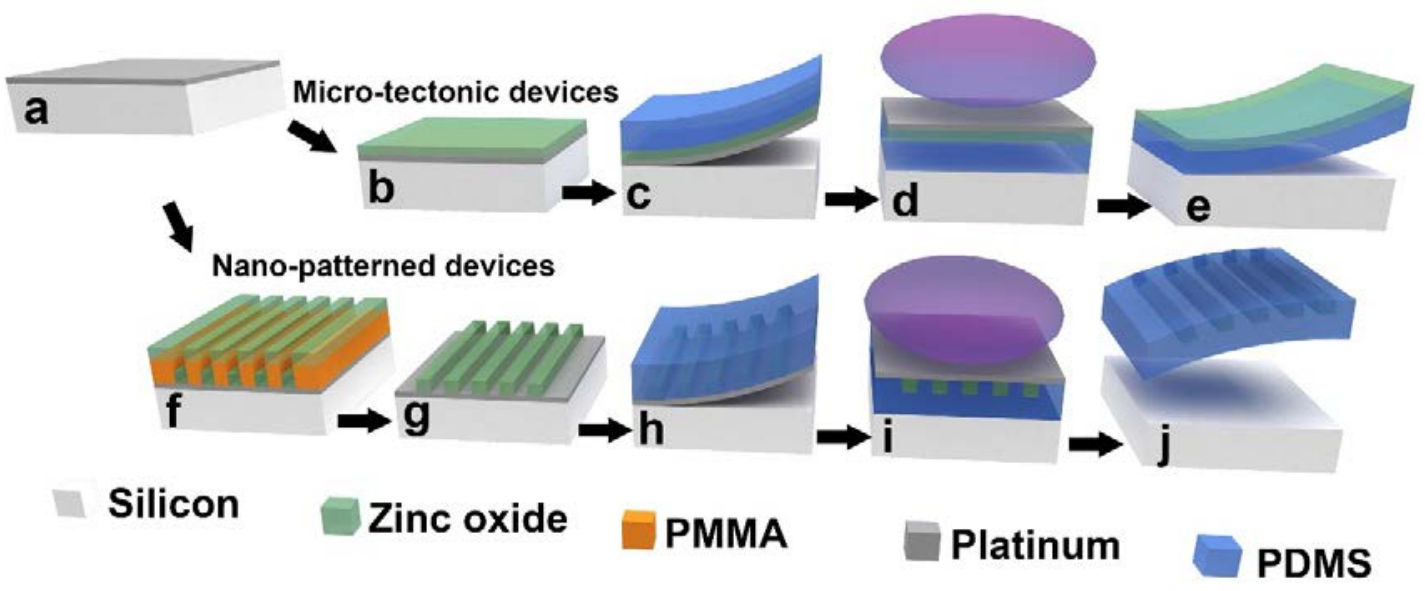

Figure 1. Realization of transparent, stretchable devices. Approaches for fabrication of both large area micro-tectonic and nano-patterned devices are schematically depicted. (a) A platinum layer $50 \mathrm{~nm}$ thick, with no adhesion promoter, is deposited on a silicon wafer. (b) Oxygen-deficient ZnO thin film are sputter-deposited at $250{ }^{\circ} \mathrm{C}$. (c) PDMS is spun-on and cured, with the layers then peeled off the silicon wafer. (d) The platinum layer is removed by reactive ion etching. (e) The completed microtectonic device is released from the wafer. (f) Nano-patterned devices commence with an electronbeam lithography step to define features in PMMA on top of which zinc oxide thin films are deposited by sputtering. (g) Excess ZnO is lifted-off in acetone. (h) Similar to (c) PDMS is spun-on, cured, and removed with embedded nanostructures from the silicon wafer. (i) The platinum layer is removed by reactive ion etching. (j) The completed nano-patterned device is released from the wafer.

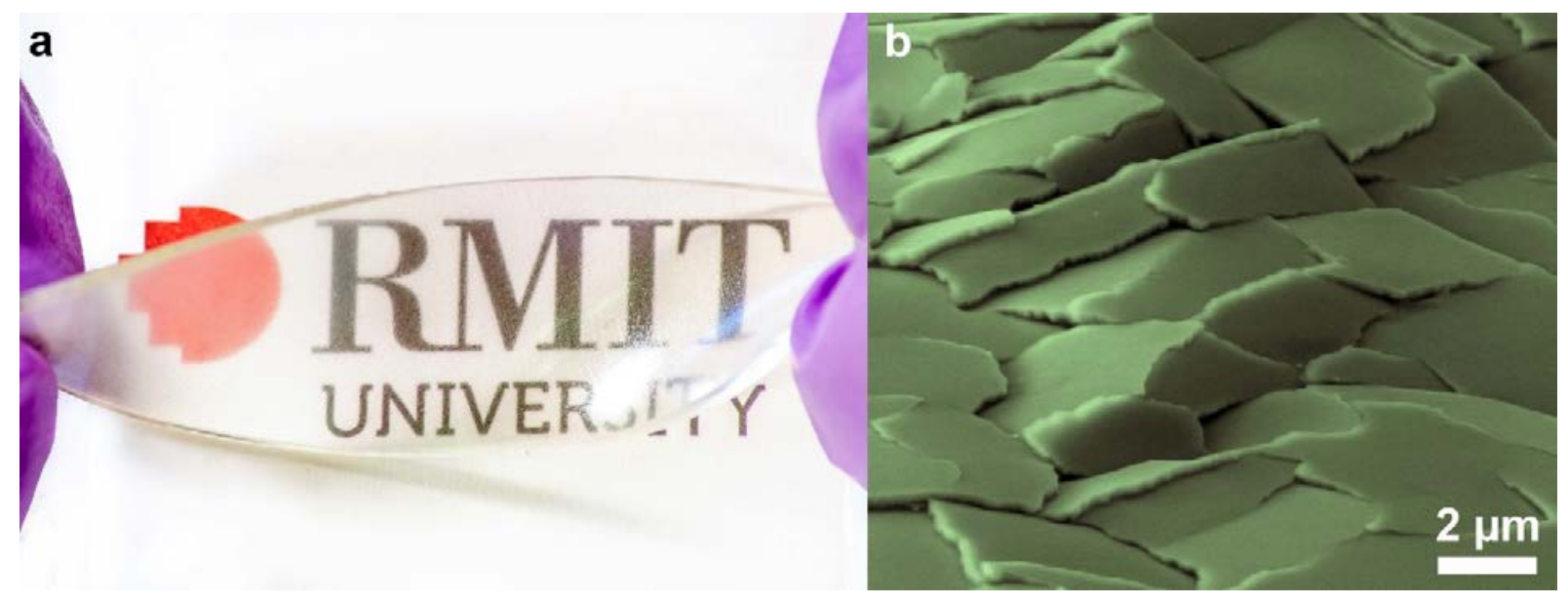

Figure 2. (a) Transparency and the ability to flex and twist a micro-tectonic $\mathrm{ZnO}$ device is shown in this photograph. (b) False-colour scanning electron micrograph of the surface-cracked micro-tectonic surface of $\mathrm{ZnO}$. 


\section{WILEY-VCH}

The micro-tectonic phenomena governs the stretchability of the thin oxide films. This phenomena occurs when thin, brittle oxide film are incorporated into elastomeric films, the brittle oxide layer forms micrometre-sized plates which overlap and slide over each other (Figure 2b) - this was a phenomenon observed previously in the case of indium tin oxide (ITO) stretchable electronics. ${ }^{[11]}$ Due to the high adhesion to the elastomer, an electrical contact is maintained between individual plates which combine to form one large functional surface.

\section{Efficient $\mathrm{H}_{2}$ and $\mathrm{NO}_{2}$ Sensing at Room Temperature}

Gas sensors fabricated on flexible platforms that are capable of operating at room temperature are still in their infancy. ${ }^{[12]}$ Most gas sensors, even on rigid platforms operate at elevated temperatures of several hundred degrees Celsius. ${ }^{[13]}$ Moreover, nanowire-based gas sensors, capable of operating at room temperature ${ }^{[14]}$ present integration challenges with the existing thin film technologies.

Oxygen-deficient ZnO films with a micro-tectonic surface morphology were defined with electrode pairs and operated as conductometric sensors. The oxygen-deficient nature of the $\mathrm{ZnO}$ allows for higher adsorption of the test gases (hydrogen and nitrogen dioxide) which directly translates to superior sensitivity. ${ }^{[15,16]}$ The electrical resistance characteristics of the micro-tectonic ZnO/PDMS sensor were acquired in situ under sequential exposure to zero air, hydrogen, and nitrogen dioxide (Figure 3a). Such a sequence of exposure allows the study of the dynamic response of the sensors towards a reducing and an oxidizing gas in a single exposure cycle. A rigid, smooth $\mathrm{ZnO}$ thin film on silicon was defined with identical electrodes and used as a comparative reference. Changes in $\mathrm{ZnO}$ resistance for the stretchable 


\section{WILEY-VCH}

devices was acquired in both relaxed and strained states, details of which are stated in the Experimental Details section.

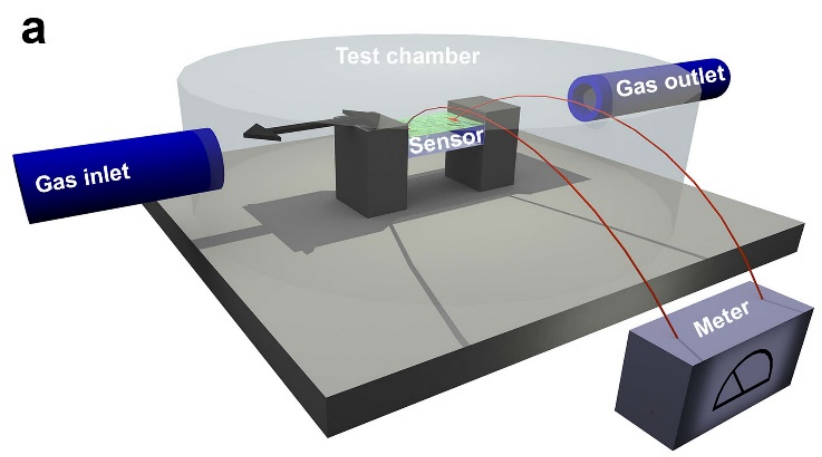

b

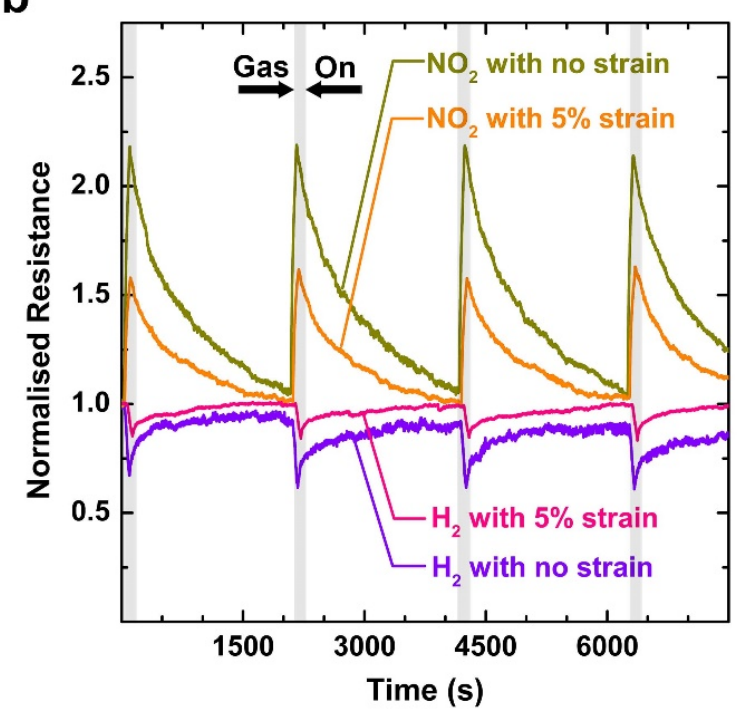

Figure 3. Testing of the $\mathrm{ZnO}$ gas sensors: (a) Schematic representation of a sample during test in a chamber with controlled gas flow and in situ resistance measurement and (b) Room temperature response of the micro-tectonic sensor to hydrogen and nitrogen dioxide in relaxed and stretched states (grey/shaded areas represent gas exposure for $80 \mathrm{~s}$ ).

The room temperature dynamic response of the micro-tectonic sensors shows a clear response to hydrogen gas. The exposure results in a rapid decrease in resistance and an inverted exponential recovery. The sensor also shows a high converse sensitivity (resistance goes up on exposure) to nitrogen dioxide and a soft quadratic recovery (Figure 3b). Under strain (up to $5 \%$ ), a reduction in sensitivity is observed even though the response characteristic is 


\section{WILEY-VCH}

maintained. The sensor shows high stability in its relaxed and strained states upon multiple exposures.

It is seen that both test gases (hydrogen and nitrogen dioxide) have opposite effects on the resistance, which can be utilized to clearly distinguish between the reducing (hydrogen) and oxidizing (nitrogen dioxide) gases. The mechanism of gas interaction with the sensing material is largely governed by the chemical properties of each material. Hydrogen is a reducing (electron-donating) gas, and hence, the resistance of $\mathrm{ZnO}$ decreases. On the other hand, a reaction with an oxidizing gas such as $\mathrm{NO}_{2}$ would cause depletion of carriers from the valence band and cause an increase in the material's resistance. This novel category of microtectonic gas sensors exhibits a response that is comparable to other high sensitivity surfaceenhanced gas sensors for hydrogen ${ }^{[14]}$ and nitrogen dioxide. ${ }^{[17]}$

The reduction in sensitivity seen on the application of strain can be attributed to the microtectonic nature of the sensor. The performance of the sensors under stress is largely governed by the effective reduction of the active area of the $\mathrm{ZnO}$ film. With increasing strain, a smaller number of micro-tectonic plates stay in contact. This results in an overall reduction in the active area of the conductometric gas sensor, resulting in an increase in the baseline resistance $\left(R_{0}\right)$ of $\mathrm{ZnO}$. Therefore, no change in the response pattern is observed, other than a reduction in sensitivity. Furthermore, the reduction in sensitivity is an indication that the overlapping ZnO plates do not show a piezotronic effect owing to their micro-tectonic morphology. ${ }^{[18-}$

${ }^{21]}$ Such an effect is also observed and described by Gutruf et al., ${ }^{[11]}$ where the resistance of ITO micro-tectonic plates changed similarly under applied strain.

To benchmark the gas sensitivity of the $\mathrm{ZnO}$ micro-tectonic surface used in this study, we fabricated a rigid analogue $(\mathrm{ZnO} / \mathrm{Si})$ using identical fabrication parameters. Owing to the 


\section{WILEY-VCH}

considerably lower sensitivity of the rigid analogue, an elevation in operating temperature and a larger gas exposure time were required to obtain a measurable response from the rigid analogue. As such, both the micro-tectonic sensor and its rigid analogue were tested under identical temperature and exposure conditions to ensure an accurate comparison. It is seen that the micro-tectonic sensor shows a higher sensitivity (>20\%) and a significantly faster response than the rigid analogue (Figure S1).

We believe that the increased sensitivity of the micro-tectonic sensor compared to the rigid film is caused by multiple factors. Firstly, an enhanced surface area due to the micro-tectonic morphology (Figure S2 and Figure S3) enables larger number of gas molecules to interact with the ZnO surface. ${ }^{[22]}$ Secondly, the gas permeable nature of PDMS towards both test gases further maximize the exposed area by allowing the diffusion of gas molecules through the bottom of the substrate. ${ }^{[23]}$

We believe that this transparent, lightweight, stretchable and highly sensitive micro-tectonic sensor is highly relevant for portable hazardous gas detection, due to its low energy consumption, robustness, and curvilinear adaptability. Due to their bio-compatible nature, an in vitro application such as a micro-capsule hydrogen gas sensor that can replace expensive and inaccurate hydrogen breath tests to detect food intolerances can also become feasible.

\section{Reversible Ultra-Violet Photosensitivity}

$\mathrm{ZnO}$ is unique due to its sensitivity to multiple sources of stimuli. In addition to its gas sensing properties, its photosensitive nature can be utilised for detecting ultra-violet (UV) radiation. While ZnO-based UV sensors on rigid substrates are extensively reported, ${ }^{\text {[24-30] }}$ stretchable and transparent UV sensors have not been explored. As such, we investigated the 


\section{WILEY-VCH}

UV sensing properties of our $\mathrm{ZnO} / \mathrm{PDMS}$ stretchable devices under elongation and compression in a high resolution stretching apparatus (Figure 4a).
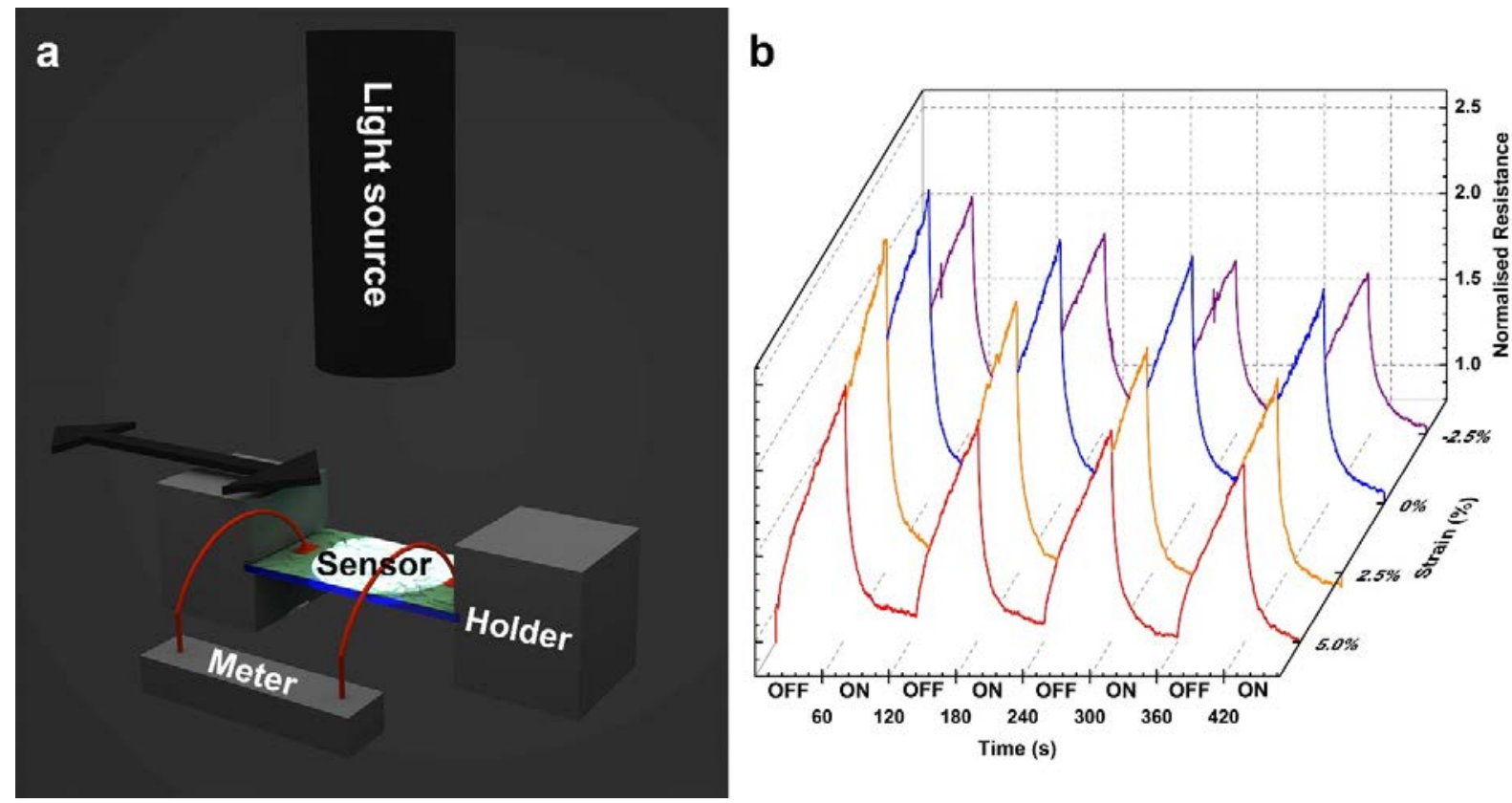

Figure 4. Optical sensing response of micro-tectonic $\mathrm{ZnO}$ to ultra-violet radiation. (a) Schematic of testing arrangement with high energy UV illumination and micro-scale stretching capability for in situ measurements. (b) Normalized resistance under cyclic exposure to darkness (OFF state) and broadband UV illumination of 1 sun (ON state) for different levels of uniaxial strain.

Oxygen-deficient $\mathrm{ZnO}$ films with a micro-tectonic surface morphology were defined with electrode pairs and operated as conductometric sensors, in a configuration identical to the gas sensors described above. The dynamic response of resistance of the sensor subject to cyclic exposures of UV light is depicted in Figure 4b. Upon exposure to UV light, the resistance of the device decreases. We refer to the device state under UV exposure as ON, while the ambient light state is termed OFF. The resistance in the OFF state is dependent on the intensity of ambient light and can result in an open circuit measurement in absolute darkness. On the other hand, the ON state is highly stable, and is therefore used as the steady state. As such, we normalized the all resistance values to the ON state resistance. This normalisation is 


\section{WILEY-VCH}

necessary to ensure an accurate comparison between the sensors at varying strain states owing to their different initial resistance values.

The devices were tested both in a relaxed as well as mechanically strained state. In a relaxed state (no strain) and given a limited recovery time of $60 \mathrm{~s}$ an average OFF/ON ratio of 2.25 is consistently observed. To further assess the device performance, we allowed a larger recovery period of $900 \mathrm{~s}$. It is seen that the OFF/ON ratios are significantly higher (up to 100) (Figure S4). Under compression and strain of up to $2.5 \%$, little variation in performance is seen. On being stretched beyond 2.5\%, a slight decrease in OFF/ON ratios can be observed. (Figure $4 \mathrm{~b}$ ). This can be ascribed to the disconnection of some $\mathrm{ZnO}$ micro-tectonic plates on being forced apart due to the applied strain. This creates a gradual build-up of non-conductive areas, causing an increase in resistance, to a point where the film eventually becomes insulating, leading to an open circuit measurement. ${ }^{[11]}$ Effectively, this leads to a reduction in the active sensing area which explains the drop in performance once stretched. Similarly, the UV sensor also showed a slightly deteriorated response under compression, which can be attributed to a decrease in exposed surface area, as well as bowing of the PDMS substrate, and as a result the $\mathrm{ZnO}$ plates. The nearly linear recovery along with a cubic response to UV light is comparable to nanoparticle ${ }^{[24]}$ or nanowire ${ }^{[31]}$ based sensors reported elsewhere, which indicates the sensor performance is largely governed by the surface morphology. However, when compared to strained nanowire $\mathrm{ZnO}$ sensors it becomes evident that a better performance under strain can be achieved with micro-tectonic devices. ${ }^{[32]}$ 


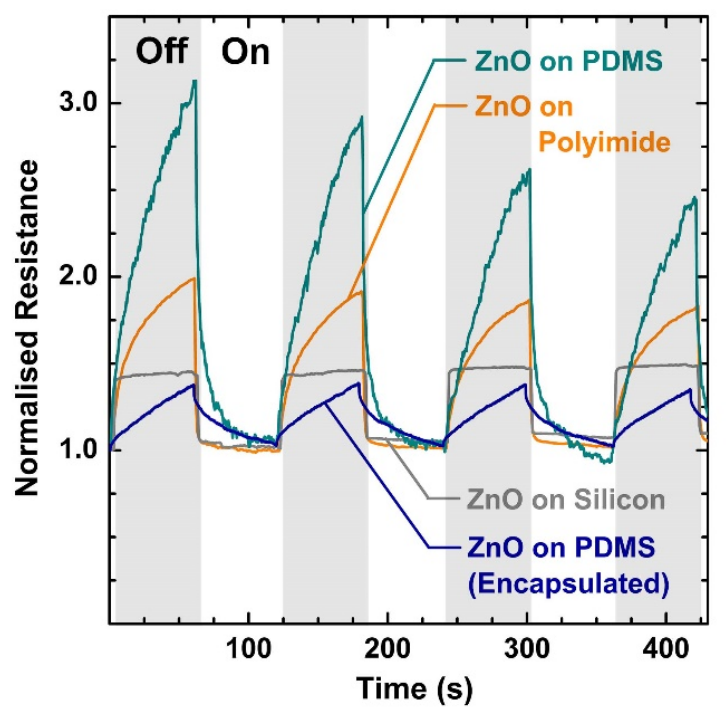

Figure 5. Sensitivity comparison of surface engineered ZnO-UV sensors. UV sensitivity of ZnO sensors fabricated on silicon, polyimide and transferred to PDMS with identical synthesis parameters.

To effectively benchmark the performance of our flexible sensor, we also tested the UV sensitivity of a bendable $\mathrm{ZnO} /$ Polyimide and $\mathrm{ZnO} / \mathrm{Si}$ rigid analogue (Figure 5).

We explain the high sensitivity of the micro-tectonic sensors using the following mechanism that drives a reversible change in resistance. The oxygen-deficient nature of the $\mathrm{ZnO}$ films used in this study attracts ambient oxygen, resulting in its adsorption on to the oxide surface. The electrical conduction decreases when oxygen is absorbed from ambient air, resulting in the formation of a depletion layer on the $\mathrm{ZnO}$ surface (OFF state). When exposed to UV light, the photogenerated carriers driven by the resulting electric field move to the surface, where they neutralize the absorbed oxygen leaving behind unpaired electrons which result in the increased conductivity (ON state). ${ }^{[31]}$ The aforementioned process is further facilitated by the micro-tectonic morphology of the $\mathrm{ZnO}$, which provides a larger surface area for the oxygen adsorption, and hence, a greater proportion of unpaired carriers. This effect results in enhanced OFF/ON ratios seen in our devices. 


\section{WILEY-VCH}

To further investigate the influence of surface morphology on the sensitivity, we prepared a sensor through direct deposition of $\mathrm{ZnO}$ on polyimide, with identical process parameters. The resulting surface topology shows a higher roughness than the silicon-based sensor but lower than the PDMS based sensor (Table 1). Scanning electronic micrographs and surface roughness/morphology profiles comparing the surfaces are present in Supplementary Information (Figures S2 and S3, respectively). These films maintain a high degree of crystallinity as verified by X-ray diffraction (Figure S5).

Table 1: Average surface roughness $R_{\mathrm{a}}$ of $\mathrm{ZnO}$ on the different sensing substrates.

\begin{tabular}{cc}
\hline Substrate & $\begin{array}{c}\text { Average Surface } \\
\text { Roughness } \boldsymbol{R}_{\mathbf{a}}(\mathbf{n m})\end{array}$ \\
\hline Silicon & 6.08 \\
Polyimide & 43.26 \\
PDMS & 160.29 \\
\hline
\end{tabular}

The performance of the polyimide-based sensors show an inverted cubic recovery with a sharp response to UV exposure (Figure 5), albeit with a lower sensitivity compared to the PDMS-based sensor. This clearly indicates that a rough surface provides more surface area for oxygen adsorption, and therefore, the change in resistance is higher on UV exposure. The effect of surface roughness on the sensing performance is clearly highlighted from (Figure 5 and Table 1). It is observed that a higher surface roughness results in a greater sensitivity. As such, the PDMS-based sensor outperforms the other devices. This hypothesis is further tested by encapsulating the $\mathrm{ZnO} / \mathrm{PDMS}$ sensor to minimize the rate of oxygen adsorption on the ZnO surface. As expected, the sensitivity drops significantly (Figure 5). When given sufficient recovery time, the encapsulated sensor still shows an OFF/ON ratio of 16 which is due to the oxygen permeable nature of PDMS which allows the ambient oxygen to gradually diffuse (Figure S4). Furthermore, we characterised the response speed of the sensors 


\section{WILEY-VCH}

(Table S2), to enable a direct comparison with literature. It can be seen that the OFF speed of the sensors on polymeric substrates is slower than its rigid counterpart while the ON speed outperform the rigid sensors consistently. In order to eliminate the possibility of the crystal structure alterations affecting the behaviour of the micro-tectonic films, measurements under elevated temperatures were acquired. Elevated temperatures induce a thermal expansion mismatch between the oxide thin film and PDMS stressing the oxide film, thereby causing a change in crystal structure. ${ }^{[33]}$ No obvious change in response to UV light is observed at temperatures of up to $150{ }^{\circ} \mathrm{C}$ degrees (Figure S6). This is mainly because the micro-tectonic plates slide over each other under strain, thereby preventing a thermal stress induced band shift. It is therefore apparent that the higher sensitivity of the ZnO/PDMS sensor is largely attributed to the unique high roughness surface structure. This high temperature measurement to validate our initial hypothesis also highlights the thermal stability of micro-tectonic sensors.

We also conducted transmission measurements were carried out to ascertain the spectral sensitivity of the sensor. A strong absorbance peak between 290 and $400 \mathrm{~nm}$ (Figure S7) is observed. This implies that the ZnO-based micro-tectonic sensor is UV sensitive in a range of 290 to $400 \mathrm{~nm}$. The spectral sensitivity of the sensor lies directly in the UV-A and UV-B bands, ${ }^{[34]}$ which can be harnessed to detect the most harmful type of UV light and can prove crucial for the prevention of skin aging, eye damage, and skin cancer.

As such, we have demonstrated a micro-tectonic-based high performance, flexible UV sensor which has physical characteristics such as conformability, low weight, transparency, and stretchability making it favourable for applications. Additionally, we have provided evidence that the micro-tectonic nature of the sensor is highly beneficial for sensing applications by offering enhancement in the active and responsive functional oxide area. 


\section{WILEY-VCH}

\section{Mechanically Tunable Diffraction Gratings}

The ability to realize sub-micron ZnO structures on a flexible PDMS substrate provides an opportunity to exploit their characteristics in the optical domain as well. One class of optical components often requiring sub-micron features are diffraction gratings which are already found a wide of range of applications in telecommunication ${ }^{[35]}$ and spectroscopy. ${ }^{[36]}$ The use of a flexible substrate, such as PDMS, offers the potential for tuning the grating's period and diffraction properties via mechanical deformation. ${ }^{[37]}$

Our novel fabrication technique allows the realization of tunable diffraction gratings that can be periodic in both dimensions and incorporate oxides like ZnO. Therefore, we designed $\mathrm{ZnO} / \mathrm{PDMS}$ diffraction gratings to demonstrate surface strain sensing with a high degree of accuracy without considerable mechanical impact on the underlying elastomeric substrate. $\mathrm{ZnO}$ was deposited onto an electron beam lithographically defined structure, and patterned by a 'lift-off' process. The device comprised of a $\sim 2 \mathrm{~mm}$ thick PDMS substrate with an embedded $\mathrm{ZnO}$ grating with a short grating period of $1 \mu \mathrm{m}$ and an overall size of $125 \mathrm{~m}^{2}$. A ZnO film thickness of $70 \mathrm{~nm}$ was used to introduce the minimum possible high modulus material to minimize mechanical impact on the substrate. The experimental setup is composed of a high resolution stretching stage in which the device is clamped to allow for precise deformation. A laser is aimed at the grating which casts a first order diffraction pattern onto a semi-transparent screen with calibrated marks for an accurate diffraction angle measurement

\section{(Figure 6a).}




\section{WILEY-VCH}

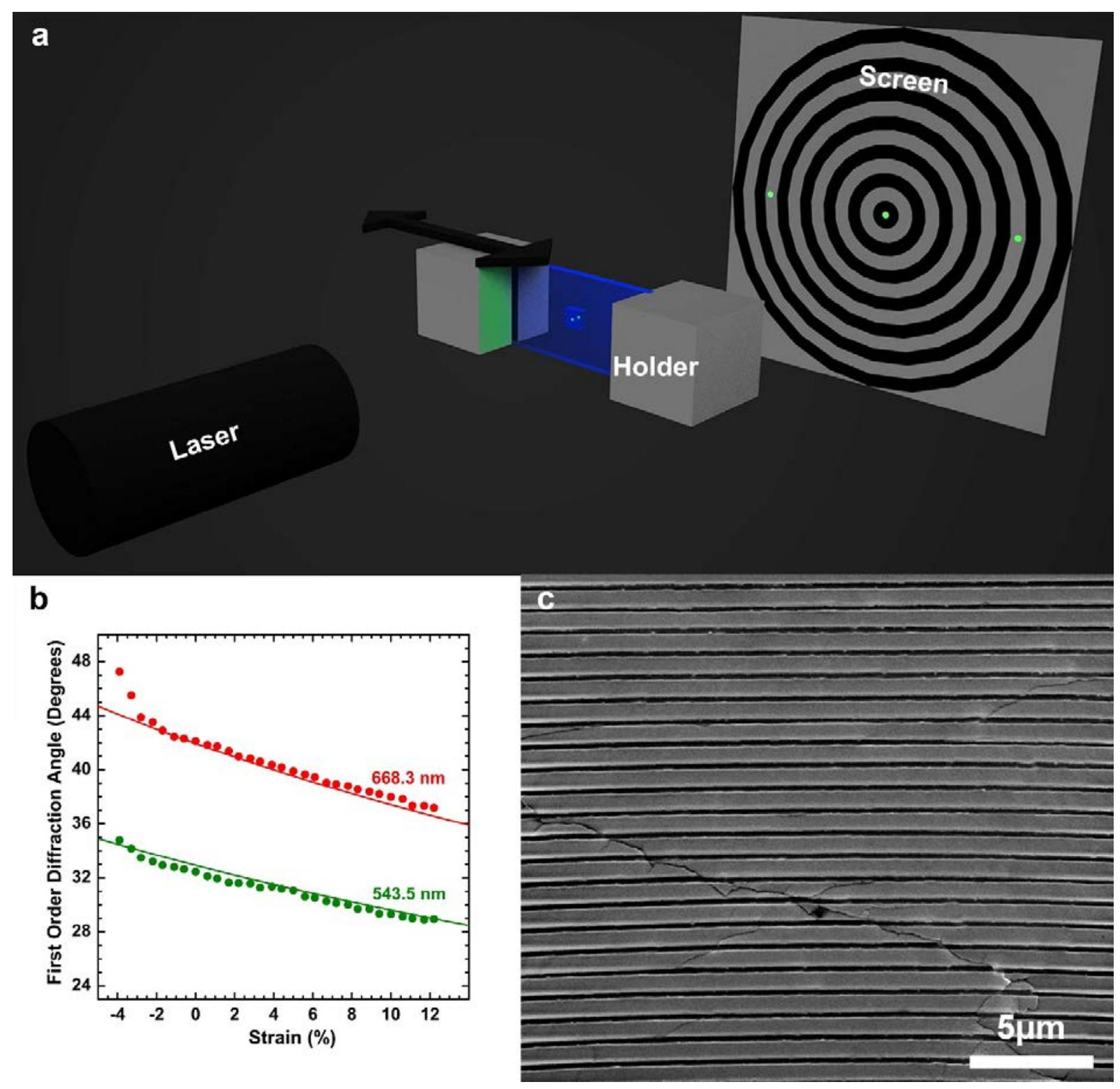

Figure 6. Testing of the stretchable $\mathrm{ZnO}$ gratings. (a) Optical setup with a laser radiating at the gratings with a screen at a fixed length to visualize the first order diffraction angle which are imaged from the back of the screen for analysis. (b) Changes in first order diffraction angle with mechanical strain at two laser wavelengths. The data markers indicate measurements, while the continuous line represents an analytical fit based on equation (2). (c) Electron micrograph of the $\mathrm{ZnO}$ gratings after extensive testing.

The measurement was executed by applying negative and positive strain ranging from $-4 \%$ to $12 \%$, while the diffraction angle was observed in situ. The data markers in Figure 6b display the recorded diffraction angles for green and red laser illumination. The measured diffraction angles are in excellent agreement with the prediction based on the grating equation, presented 


\section{WILEY-VCH}

in the Experimental Details section. When the sample is compressed, the measured angles slightly deviate from the model, in particular for red light. This deviation is related to the bending of the PDMS film under compression, with the non-planar structure not considered by the analytical expression.

To evaluate the device performance and mechanical robustness after rough handling the grating was tested after being rotated by $90^{\circ}$ so that the strain impacts perpendicular to the grating period and a strain of $-4 \%$ to $12 \%$ was applied. As expected, only a slight change in diffraction angle was observed, which is caused by the intrinsic Poisson's ratio of PDMS ( 0.5). Figure 6c shows an SEM micrograph of a fabricated diffraction grating after extensive stretching in the direction of the grating period and perpendicular to it. Apart from some minor cracks, the gratings structure remained largely intact, due to the strong adhesion to the PDMS substrate. The gratings remain fully functional even after the small cracks occur due to the oxide being embedded in the PDMS. Therefore, the significant refractive index contrast (PDMS 1.4, $\mathrm{ZnO} \sim 2.0$ ) is still intact resulting in no degradation of device performance.

Considering a possible detection of $0.1^{\circ}$ change of the diffraction angle, in our simple measurement setup, the measurable change in strain is in the order of $0.26 \%$ and $0.36 \%$ for green light and $0.18 \%$ to $0.26 \%$ for red light over a strain range of $-2 \%$ to $12 \%$. In absolute terms, this equals to a change in period of $2.1 \mathrm{~nm}$ to $4.0 \mathrm{~nm}$ for green light and $1.8 \mathrm{~nm}$ to $2.9 \mathrm{~nm}$ for red light. In the tested device, the stretchable area is limited to 125 grating periods, allowing the measurement of absolute strain of $~ 300 \mathrm{~nm}$ using the green laser and $~ 250 \mathrm{~nm}$ when using the red laser. The performance of the fabricated devices agrees well with the calculated results and allows for surface strain measurement in the nanometre regime with little impact on the elastomeric substrate. 


\section{WILEY-VCH}

\section{Conclusions}

In summary, we have demonstrated $\mathrm{ZnO}$-based gas, UV, and strain sensing on a stretchable platform, where the functional material is stretchable itself. It has been shown that our stretchable sensors outperform their rigid counterparts and do not require elevated temperatures for efficient performance. The unique micro-tectonic structure of the functional $\mathrm{ZnO}$ films has been shown to result in enhanced sensing performance of the stretchable templates. Such transparent, stretchable $\mathrm{ZnO}$ devices offer significant potential for the development of cost-effective, bio-compatible, functional, and curvilinear electronic and optoelectronic devices. Furthermore, a precise control over spatial resolution in the nanometre regime is shown, allowing for applications such as oxide-based gratings, which exhibit high accuracy and can track surface movements of PDMS down to sub-10 nm resolution. The demonstrated devices will pave the way for more robust stretchable electronics that do not require a rigid island approach due to the stretchable nature of the functional element.

\section{Experimental Details}

\section{Device fabrication}

The fabrication of the transparent PDMS-based $\mathrm{ZnO}$ devices relies on the platinum transfer technique described in detail in Gutruf et al. ${ }^{[11]}$ The simplified production process is depicted in Figure 1. The process starts with a platinum layer deposited by electron beam evaporation on a standard cleaned silicon wafer (Figure 1a). Subsequently, $500 \mathrm{~nm} \mathrm{ZnO} \mathrm{thin} \mathrm{films} \mathrm{with} \mathrm{a}$ preferential (002) crystal orientation were deposited on $\mathrm{Pt} / \mathrm{Si}$ substrates from a pure zinc (99.99\%) target via reactive DC magnetron sputtering at a temperature of $250{ }^{\circ} \mathrm{C}$. Detailed sputtering conditions are presented as Supplementary Information (Table S1). The oxygen content in sputtered $\mathrm{ZnO}$ thin films is controlled by altering the oxygen partial pressure during the sputtering process. ${ }^{[38,39]} \mathrm{N}$-type conduction behaviour of the films was verified with the 


\section{WILEY-VCH}

hot point probe method. ${ }^{[40]}$ Furthermore, the crystallinity was verified by XRD analysis (Figure S4). To verify the oxygen-deficient nature of the sputtered film an XPS analysis was carried out (Figure S8). The $\mathrm{ZnO} / \mathrm{Pt}$ sandwich was then removed from its rigid carrier by casting PDMS (2 mm) onto the $\mathrm{ZnO}$ layer with a subsequent hot plate cure at $120^{\circ} \mathrm{C}$ for 8 min and an immediate peel-off (Figure 1c). The wafer-sized ZnO/PDMS device was flipped over onto a silicon carrier and the Pt layer was then removed by reactive ion etching (7.5 min, $100 \mathrm{~W}$ in argon atmosphere at 70 mTorr working pressure) leaving the $\mathrm{ZnO}$ exposed (Figure 1d). The devices for the gas sensing and photosensitivity test were then carefully diced into $10 \mathrm{~mm} \times 20 \mathrm{~mm}$ specimens.

The fabrication of the nanoscale gratings occurs similar to the fabrication of the unpatterned devices, except for the additional lithography process. A $250 \mathrm{~nm}$ thick polymethylmethacrylate (PMMA) layer is applied to the platinum-coated silicon wafer via spin coating followed by a hard bake at $180{ }^{\circ} \mathrm{C}$. The electron beam sensitive PMMA is then exposed with an electron beam lithography system (Nabity EBL system on a FEI Nova SEM equipped with a field emission gun) writing the negative mask of the diffraction grating in a serial process. With a subsequent immersion in a MIBK developer to wash away the exposed regions of the transmission diffraction grating, the sample is then placed in the reactive sputterer to deposit $70 \mathrm{~nm}$ thick $\mathrm{ZnO}$ films at room temperature, in a 6:4 gas ratio of $\mathrm{Ar} / \mathrm{O}_{2}$ (Figure 1f). Lift-off is then performed in an acetone bath removing the excess $\mathrm{ZnO}$. Subsequently the finished gratings were heated to $400{ }^{\circ} \mathrm{C}$ for $1 \mathrm{~h}$ under oxygen atmosphere to render them transparent (Figure 1g). Analogous to the non-patterned $\mathrm{ZnO}$ device production, PDMS was cast and cured on the gratings and then removed by peeling (Figure 1h). Again, the platinum layer was removed by reactive ion etching (Figure 1i) exposing the $\mathrm{ZnO}$ diffraction pattern. The devices where then removed from the carrier and diced into $30 \mathrm{~mm} \times 50 \mathrm{~mm}$ pieces. 


\section{WILEY-VCH}

\section{Gas sensitivity testing}

Gas sensitivity testing was conducted in a temperature controlled chamber (Linkam HFS600E-P with Linkam T95-PE controller) with gas flow control (MKS Multigas Controller 647B) (Fig. 3(a)). The flexible $\mathrm{ZnO}$ gas sensing device was mounted on the temperature controlled chuck and heated to $100{ }^{\circ} \mathrm{C}$. The resistance was measured in situ via copper pads of $0.5 \mathrm{~cm}^{2}$ in area, placed carefully at a distance of $10 \mathrm{~mm}$ on the $\mathrm{ZnO}$ surface, held down by micro probes for both rigid $\mathrm{ZnO}$ and micro-tectonic surfaces. The room temperature measurements were conducted in a similar manner. The exposure of hydrogen $\left(1 \% \mathrm{H}_{2}\right.$ in zero air balance with a flow rate of 200 sccm) was followed by an exposure to zero air with a flow rate of $800 \mathrm{sccm}$ to monitor the response speed of the sensor. The $\mathrm{NO}_{2}$ testing was executed in a similar manner to the hydrogen sensing tests with $\mathrm{NO}_{2}$ exposure $\left(9.9 \mathrm{ppm}\right.$ of $\mathrm{NO}_{2}$ in zero air balance) with a flow rate of $200 \mathrm{sccm}$, followed by a flow rate of $800 \mathrm{sccm}$ of zero air.

The room temperature measurements were taken with a zero air exposure of $35 \mathrm{~min}$ and a subsequent $80 \mathrm{~s}$ exposure to hydrogen and $\mathrm{NO}_{2}$, respectively. The resistance measurements at elevated temperature were then acquired every second (Figure S1) with an exposure of 35 min zero air and a subsequent 10 min hydrogen exposure. All gas sensing measurements were taken under a steady ambient illumination.

\section{Photosensitivity testing}

Photosensitivity testing was conducted with a broadband light source (Abet Technologies Model LS150) as shown in Figure 4a, and the shutter was operated manually in a 1 min dark/exposure cycle. The $\mathrm{ZnO}$ sensor was clamped on to a custom-made stretching stage with a displacement resolution of $2.5 \mu \mathrm{m}$. Subsequently, testing was conducted (Fig. 4(d)) under compression and elongation while monitoring the resistance in situ via bonded electrodes using gold ribbon and silver bonding epoxy. 


\section{WILEY-VCH}

\section{Diffraction angle measurement}

The angle of the diffracted light was predicted based on the grating equation for normal incidence according to:

$$
\sin (\theta)=\frac{m \lambda}{\Lambda}
$$

where $\theta$ is the diffracted angle, $m$ the diffraction order, $\lambda$ the operating wavelength, and $\Lambda$ the period of the diffraction grating. The period of the diffraction grating was designed to be $1 \mu \mathrm{m}$ in order to allow 1st order diffraction for green and red laser sources. The green and red solid lines in Figure $6 \mathrm{~b}$ show the calculated diffraction angle of a $1 \mu \mathrm{m}$ period grating under strain ranging from $-5 \%$ to $13 \%$ and illuminated with green $(\lambda=543.5 \mathrm{~nm})$ and red $(\lambda=668.3 \mathrm{~nm}$ ) laser light, respectively. As strain is increased, diffraction is predicted to drop from $35^{\circ}$ to $27^{\circ}$ for green light and $45^{\circ}$ to $34^{\circ}$ for red light.

In order to test the performance of the fabricated gratings, the diffraction angle was measured using a setup as depicted in Figure 6a. A He-Ne laser (Uniphase 1974P at $543.5 \mathrm{~nm}$ ) and a diode laser (Thorlabs LDM670 at $668.3 \mathrm{~nm}$ ) were used to generate coherent green and red light, respectively. The wavelength is crucial for the accuracy of the system and was monitored by a spectrometer (Figure S9). The laser source was directly aimed at transmission grating which was mounted in stretching stage. At a distance of $10 \mathrm{~cm}$ past the grating, a semi-transparent paper screen was installed. Behind the screen, a camera (Canon 550D) was placed to capture the images of the diffracted laser beams as they illuminate the screen (Figure S10). The strain was varied from $-4 \%$ to $12 \%$ of strain with $0.25 \%$ increments.

The location of the 0th and 1st order diffracted beams can be identified as bright spots on the screen. The diffraction angle $\theta$ can be determined using a trigonometric relation: 


$$
\tan (\theta)=\frac{a}{b}
$$

where $a$ is the distance between the bright spot of 0th and 1st order diffracted beams on the screen and $b$ is the known distance of the grating to the screen.

\section{Supporting Information}

Supporting Information is available from the Wiley Online Library or from the author.

\section{Acknowledgements}

PG acknowledges an Australian Government Endeavour International Postgraduate Research Scholarship. SS and MB acknowledge Australian Post-Doctoral Fellowships from the Australian Research Council through Discovery Projects DP110100262 and DP1092717, respectively. The authors acknowledge the facilities and technical assistance of the Australian Microscopy and Microanalysis Research Facility at the RMIT Microscopy and Microanalysis Facility at RMIT University.

Received: ((will be filled in by the editorial staff))

Revised: ((will be filled in by the editorial staff)) Published online: ((will be filled in by the editorial staff))

[1] H. J. Chung, M. S. Sulkin, J. S. Kim, C. Goudeseune, H. Y. Chao, J. W. Song, S. Y.

Yang, Y. Y. Hsu, R. Ghaffari, I. R. Efimov, Advanced healthcare materials 2014, 3,

[2] S.-W. Hwang, H. Tao, D.-H. Kim, H. Cheng, J.-K. Song, E. Rill, M. A. Brenckle, B.

Panilaitis, S. M. Won, Y.-S. Kim, Science 2012, 337,

[3] H. Yan, Z. Chen, Y. Zheng, C. Newman, J. R. Quinn, F. Dötz, M. Kastler, A.

Facchetti, Nature 2009, 457,

[4] G. Gustafsson, Y. Cao, G. Treacy, F. Klavetter, N. Colaneri, A. Heeger, Nature 1992, 357 ,

[5] G. H. Gelinck, H. E. A. Huitema, E. van Veenendaal, E. Cantatore, L. Schrijnemakers, J. B. van der Putten, T. C. Geuns, M. Beenhakkers, J. B. Giesbers, B.-H. Huisman, Nature materials 2004, 3, 


\section{WILEY-VCH}

[6] I. Yagi, N. Hirai, Y. Miyamoto, M. Noda, A. Imaoka, N. Yoneya, K. Nomoto, J.

Kasahara, A. Yumoto, T. Urabe, Journal of the Society for Information Display 2008, 16,

[7] L. Sun, G. Qin, J. H. Seo, G. K. Celler, W. Zhou, Z. Ma, Small 2010, 6,

[8] J. Kim, A. Banks, H. Cheng, Z. Xie, S. Xu, K. I. Jang, J. W. Lee, Z. Liu, P. Gutruf, X. Huang, Small 2014, 11, 906.

[9] I. F. Akyildiz, W. Su, Y. Sankarasubramaniam, E. Cayirci, Computer networks 2002, 38 ,

[10] S. Walia, S. Balendhran, H. Nili, S. Zhuiykov, G. Rosengarten, Q. H. Wang, M. Bhaskaran, S. Sriram, M. S. Strano, K. Kalantar-zadeh, Progress in Materials Science 2013, 58,

[11] P. Gutruf, C. M. Shah, S. Walia, H. Nili, A. S. Zoolfakar, C. Karnutsch, K. Kalantarzadeh, S. Sriram, M. Bhaskaran, NPG Asia Materials 2013, 5,

[12] M. C. McAlpine, H. Ahmad, D. Wang, J. R. Heath, Nature materials 2007, 6,

[13] G. Sberveglieri, Sensors and Actuators B: Chemical 1995, 23,

[14] H.-T. Wang, B. Kang, F. Ren, L. Tien, P. Sadik, D. Norton, S. Pearton, J. Lin, Applied Physics Letters 2005, 86,

[15] M. Breedon, M. Spencer, I. Yarovsky, The Journal of Physical Chemistry C 2010, 114, [16] M. Nyberg, M. A. Nygren, L. G. Pettersson, D. H. Gay, A. L. Rohl, The Journal of Physical Chemistry 1996, 100,

[17] S. T. Shishiyanu, T. S. Shishiyanu, O. I. Lupan, Sensors and Actuators B: Chemical 2005, 107,

[18] G. Hu, R. Zhou, R. Yu, L. Dong, C. Pan, Z. L. Wang, Nano Research 2014, 7,

[19] R. Zhou, G. Hu, R. Yu, C. Pan, Z. L. Wang, Nano Energy 2015, 12,

[20] C. Pan, R. Yu, S. Niu, G. Zhu, Z. L. Wang, ACS nano 2013, 7,

[21] R. Yu, C. Pan, Y. Hu, L. Li, H. Liu, W. Liu, S. Chua, D. Chi, Z. L. Wang, Nano Research 2013, 6, 


\section{WILEY-VCH}

[22] G. Heiland, Sensors and Actuators 1982, 2,

[23] M. Rezakazemi, K. Shahidi, T. Mohammadi, international journal of hydrogen energy 2012, 37,

[24] J. H. Jun, H. Seong, K. Cho, B.-M. Moon, S. Kim, Ceramics International 2009, 35,

[25] D. Zhang, Journal of Physics D: Applied Physics 1995, 28,

[26] H. Kind, H. Yan, B. Messer, M. Law, P. Yang, Advanced Materials 2002, 14,

[27] J. Qi, X. Hu, Z. Wang, X. Li, W. Liu, Y. Zhang, Nanoscale 2014, 6,

[28] Z. Bai, X. Yan, X. Chen, Y. Cui, P. Lin, Y. Shen, Y. Zhang, RSC Advances 2013, 3,

[29] P. Lin, X. Chen, X. Yan, Z. Zhang, H. Yuan, P. Li, Y. Zhao, Y. Zhang, Nano

Research 2014, 7,

[30] X. Zheng, Y. Sun, X. Yan, X. Chen, Z. Bai, P. Lin, Y. Shen, Y. Zhao, Y. Zhang, RSC Advances 2014, 4,

[31] C. Soci, A. Zhang, B. Xiang, S. A. Dayeh, D. Aplin, J. Park, X. Bao, Y.-H. Lo, D.

Wang, Nano letters 2007, 7,

[32] P. Lin, X. Yan, Z. Zhang, Y. Shen, Y. Zhao, Z. Bai, Y. Zhang, ACS applied materials \& interfaces 2013, 5,

[33] R. Ghosh, D. Basak, S. Fujihara, Journal of applied physics 2004, 96,

[34] A. Becheri, M. Dürr, P. L. Nostro, P. Baglioni, Journal of Nanoparticle Research 2008, 10,

[35] A. M. Vengsarkar, P. J. Lemaire, J. B. Judkins, V. Bhatia, T. Erdogan, J. E. Sipe, Lightwave Technology, Journal of 1996, 14,

[36] G. D. Goodno, G. Dadusc, R. Miller, JOSA B 1998, 15,

[37] T. Ma, H. Liang, G. Chen, B. Poon, H. Jiang, H. Yu, Opt. Express 2013, 21,

[38] K. Ellmer, Journal of Physics D: Applied Physics 2000, 33, R17.

[39] G. Xiong, J. Wilkinson, B. Mischuck, S. Tüzemen, K. B. Ucer, R. T. Williams, Applied Physics Letters 2002, 80, 1195. 
WILEY-VCH

[40] R. Lefever, Review of Scientific Instruments 1962, 33, 


\section{WILEY-VCH}

\section{The table of contents entry}

We integrate $\mathrm{ZnO}$ into an elastomeric platform to realize transparent stretchable sensing devices. The sensors are capable of performing room temperature gas sensing, UVsensing, and nanometer accuracy strain sensing. We enable stretchability and enhanced sensitivity through introduction of the micro-tectonic effect and nanopatterning. The sensors are tested at various strain states via in situ characterization and their performance is benchmarked against rigid counterparts.

Keyword: Stretchable $\mathrm{ZnO}$ electronics

P. Gutruf, E. Zeller, S. Walia, H. Nili, S. Sriram,* and M. Bhaskaran*

Stretchable and tunable micro-tectonic ZnO-based sensors and photonics
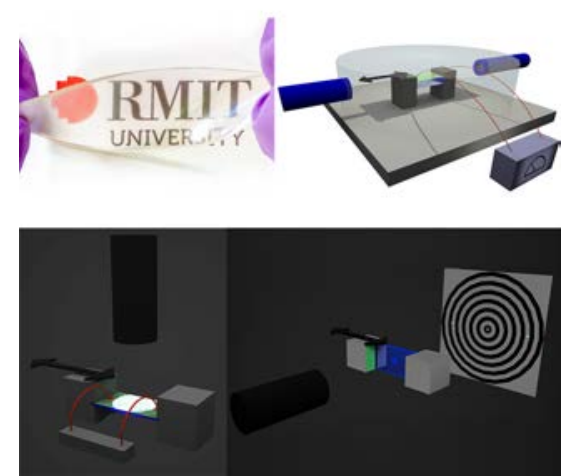